\title{
Self-Organization in Mobile Networking Systems
}

\author{
Hyun-Ho Choi, ${ }^{1}$ Seok-Chul Kwon, ${ }^{2}$ Youngwook Ko, ${ }^{3}$ and Jung-Ryun Lee ${ }^{4}$ \\ ${ }^{1}$ Department of Electrical, Electronic and Control Engineering, Hankyong National University, Anseong, Republic of Korea \\ ${ }^{2}$ Intel Corporation, Santa Clara, CA, USA \\ ${ }^{3}$ School of Electronics, Electrical Engineering and Computer Science, Queen's University Belfast, Belfast, UK \\ ${ }^{4}$ School of the Electrical Engineering, Chung-Ang University, Seoul, Republic of Korea
}

Correspondence should be addressed to Jung-Ryun Lee; jrlee@cau.ac.kr

Received 10 November 2016; Accepted 10 November 2016

Copyright (C) 2016 Hyun-Ho Choi et al. This is an open access article distributed under the Creative Commons Attribution License, which permits unrestricted use, distribution, and reproduction in any medium, provided the original work is properly cited.

Current mobile communication and networking systems, such as cellular network, wireless local area network, ad hoc network, and vehicular network, require manual configuration and centralized control for both deployment and operation. Such a paradigm would not be so feasible in future mobile networking systems, where a tremendous number of embedded devices and everyday items will be interconnected by various wireless technologies. One of the important design features for these future systems is the so-called self$\mathrm{x}$ functions including self-configuration, self-optimization, self-adaption, self-healing, and self-protection. These selforganizing functions can reduce the network deployment time, save the operational costs, enhance the network performance, and facilitate network management without human involvement. For this reason, the self-organization in networks has become an interesting research topic and still needs to be investigated for future mobile networking systems.

This special issue contains 11 papers selected from submissions to the open call for papers. These papers highlight some of the current research interests and achievements in the area of self-organizing algorithms, protocols, and applications in various mobile networking systems.

The paper by J. Park and Y. Lim is entitled "Adaptive Access Class Barring Method for Machine Generated Communications." This paper proposes an adaptive access barring method for machine type communication (MTC) in 3GPP cellular networks. Since an eNB does not know the number of MTC devices in its coverage area, it is difficult to control the barring factor by predicting the number of MTC devices in a service area of a cell. Therefore, the authors have controlled the barring factor based on the prediction of access intensity which can be measured at an eNB. Because the proposed method can manipulate the barring factor autonomously according to the access intensity, it is superior to the original method in terms of the access success probability and the collision probability.

The paper by K. Lee and J. Ko is entitled "Adaptive Power Allocation and Splitting with Imperfect Channel Estimation in Energy Harvesting Based Self-Organizing Networks." The authors have introduced an adaptive power allocation and splitting (APAS) scheme which takes imperfect channel estimations in consideration, whereas previous researches in RF-based information and energy transfer build up on the assumption that perfect channel estimation is easily achievable. The proposed APAS scheme achieves near-optimal performances for transferring energy and data over a single $\mathrm{RF}$ transmission.

The paper by J. M. Arana et al. is entitled "RandomAccess Technique for Self-Organization of 5G MillimeterWave Cellular Communications." This paper proposes two different types of random access preambles (RAPs) to reduce the processing time in the random access stage. Analyses of the correlation property, false-alarm probability, and detection probability verify that the proposed RAPs are suitable for random access in $\mathrm{mm}$ wave cellular systems with directional beams because of the smaller processing time and high detection probability in multiuser environments.

The paper by W. Wang et al. is entitled "An Adaptive Information Quantity-Based Broadcast Protocol for Safety Services in VANET." This paper proposes an information quantity-based broadcast protocol to ensure the efficiency 
of safety messages dissemination. The presented emergencydegree-based broadcast (EDCast) protocol differentiates each packet's priority for accessing the channel based on its emergency degree so as to provide vehicles with more safety information timely and accurately. In addition, an adaptive scheme is presented to ensure fast dissemination of messages in different network condition. The proposed EDCast achieves higher broadcast efficiency and less redundancy with less delivery delay so that it is feasible and necessary for incorporating information quantity of messages in designing an efficient safety message broadcast protocol.

The paper by F. Zhou et al. is entitled "Utility Maximization for Load Optimization in Cellular/WLAN Interworking Network Based on Generalized Benders Decomposition”. In this paper, a utility maximization (UTMAX) optimization model and an ASRAO algorithm based on generalized Benders Decomposition is presented to investigate load optimizing from a perspective of system utilization maximization. The authors show a tradeoff between improvements in user throughput fairness and system total throughput. Since UTMAX requires a mixed integer nonlinear programming, which is intractable intuitively, the authors proposed ASRAO to solve this problem optimally and effectively. An optional phase for expediting ASRAO is proposed by using relaxation and approximation techniques, which reduces nearly $10 \%$ iterations and time needed by normal ASRAO from simulation results. The results also show UTMAX's good effects on improving WLAN usage and edge user throughput.

The paper by J. A. Fernández-Segovia et al. is entitled "A Fast Self-Planning Approach for Fractional Uplink Power Control Parameters in LTE Networks". In this work, the authors presented the self-planning of Uplink Fractional Power Control (FPC) settings. For this purpose, the authors formulated the FPC planning problem in a cell basis through the combination of multiple regular scenarios built on a peradjacency basis. A detailed inspection on the FPC parameter values to identify the most important variables in the scenario impacting optimal FPC settings follows. And regression equations are built based on key variables for a simple FPC parameter calculation. Results show that network performance with proposed FPC parameter settings shows better performance compared with typical FPC configurations from operators.

The paper by E. Cheng et al. is entitled "A TDoA Localization Scheme for Underwater Sensor Networks with Use of Multilinear Chirp Signals." This paper takes the Multilinear Chirp (MLC) signals as the location signal to improve the anticollision ability and proposes a fast efficient detection method called Mixing Change Rate-Fractional Fourier Transform (MCR-FrFT) in order to increase the detection efficiency of MLC. The proposed method transforms the combined rates of MLC into symmetry triangle rates and then separates the multiuser signals based on the transformed rates by using FrFT. The proposed method can detect the locations signals, estimate the time difference of arrival, reduce the multiple access interference, and improve the location performance.

The paper by J. Paek et al. is entitled "A Measurement Study of BLE iBeacon and Geometric Adjustment Scheme for
Indoor Location-Based Mobile Applications." This paper performs empirical measurements on three different Bluetooth Low-Energy (BLE) iBeacon devices for indoor locationbased mobile applications. This measurement result shows that the signal strength readings from iBeacon vary significantly over different vendors, mobile platforms, environmental or deployment factors, and usage scenarios. From this measurement result, the authors proposed a simple class attendance checking application by performing a simple form of geometric adjustments to compensate for the natural variations in beacon signal strength readings.

The paper by J. Shim and Y. Lim is entitled "WSNBased Height Estimation of Moving Object in Surveillance Systems." This paper considers the physical height to differentiate an intruder from detected objects in the wireless sensor network-based surveillance system. Using the measured information from sensors, the authors estimated the height of the detected object. Based on the height, if the detected object is decided as an intruder, an alarm is given to a control center. The proposed mechanism correctly and fast estimates the height of the object without complex computation.

The paper by O. Jo et al. is entitled "Intergroup Joint Scheduling for Mitigating Asymmetric Uplink Interference in Self-Organizing Virtual Cell Networks.” This paper introduces the concept of self-organizing Virtual Cell Network (VCN). In this self-organizing VCN, the authors proposed an efficient scheduling algorithm that considers the asymmetry of interference between downlink and uplink to mitigate intercell interference with little computing overhead. The basic concept is to construct scheduling groups that consist of several users. Each user in a scheduling group is affiliated with a different cell. Then, the intercell groups are managed efficiently in the proposed VCNs. The proposed scheduling method does not require the exchange of a lot of information among base stations to schedule the users over the entire network.

The paper by W. Lee and B. C. Jung is entitled "Improving Energy Efficiency of Cooperative Femtocell Networks via Base Station Switching Off." This paper proposed BS switching-off technique for a cooperative femtocell network in which multiple femtocell BSs (FBSs) simultaneously send packets to the same mobile station. The authors formulated the optimization problem to find the optimal set of FBSs to be turned off and proposed a suboptimal scheme operating in a distributed manner in order to reduce the computational complexity of the optimal scheme. Results showed that the energy consumption is reduced compared with conventional schemes and the suboptimal scheme achieved the nearoptimal performance with lower computational complexity.

\section{Acknowledgments}

The guest editors would like to thank the authors for their contributions to this special issue and many experts who have provided constructive comments to the authors to improve the quality of the papers. Hyun-Ho Choi
Seok-Chul Kwon
Youngwook Ko
Jung-Ryun Lee 

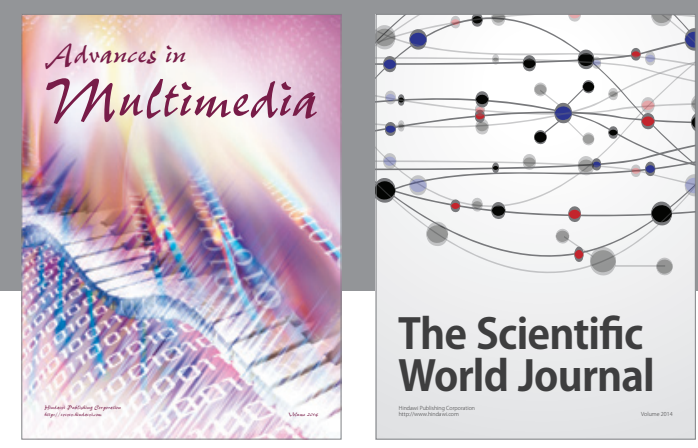

The Scientific World Journal
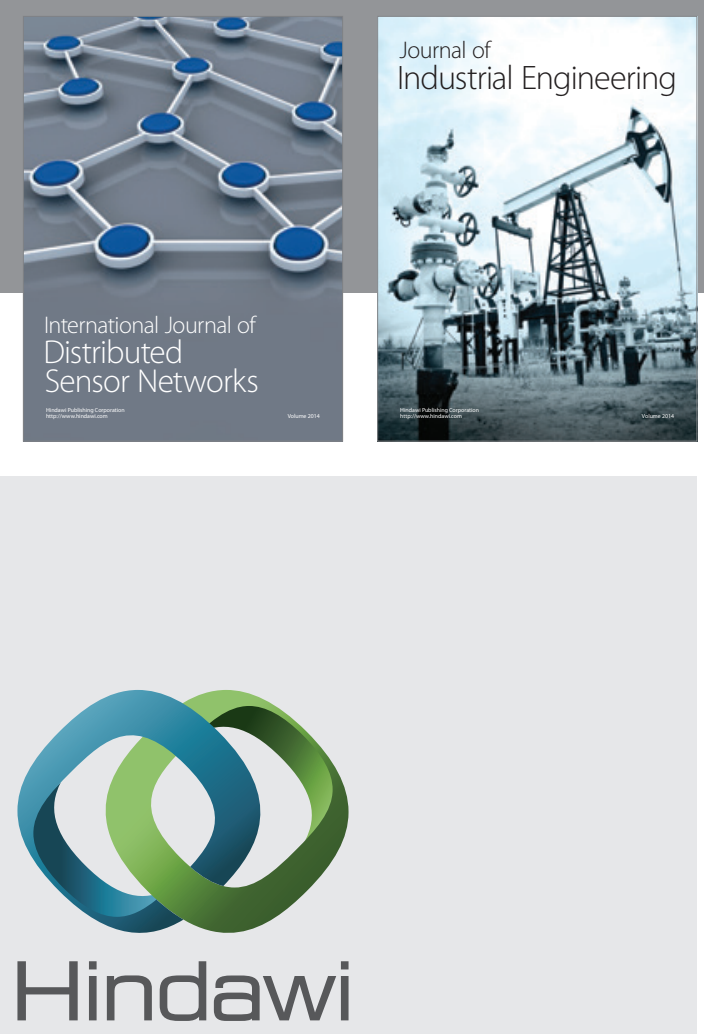

Submit your manuscripts at

http://www.hindawi.com

\section{Computer Networks} and Communications
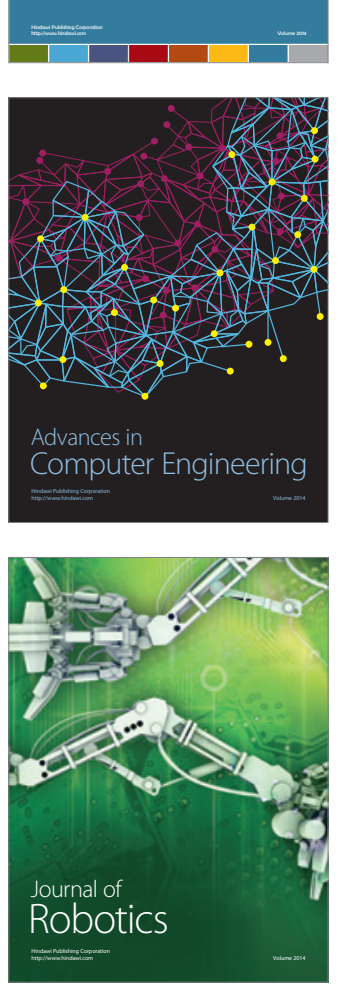
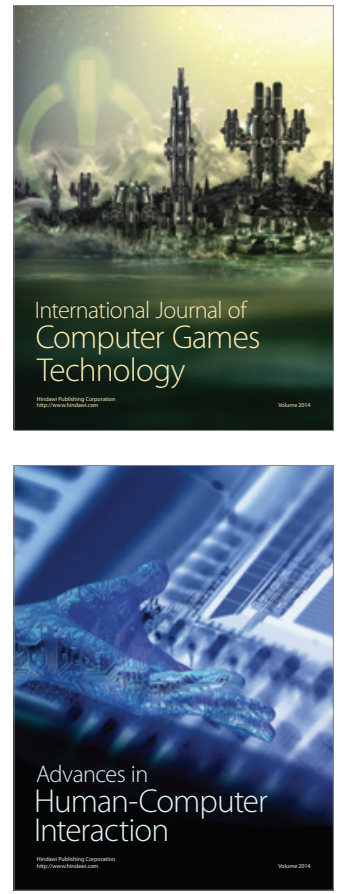
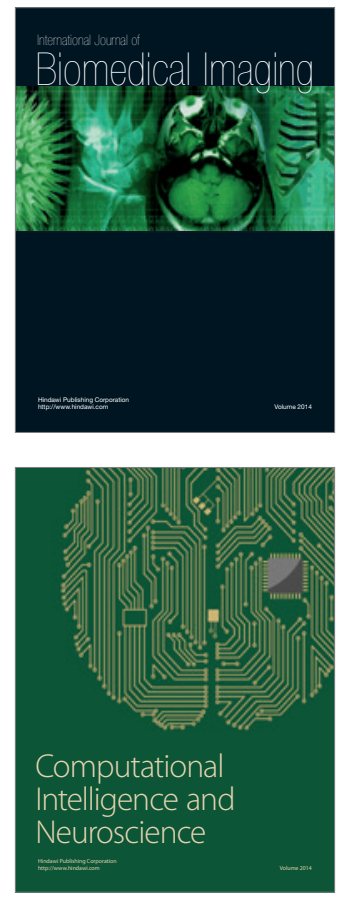
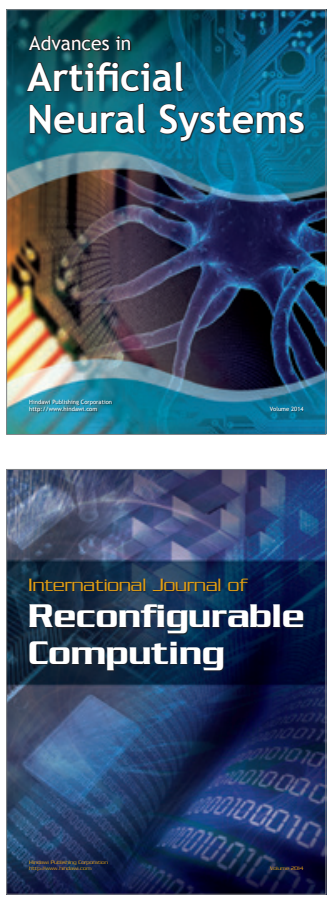
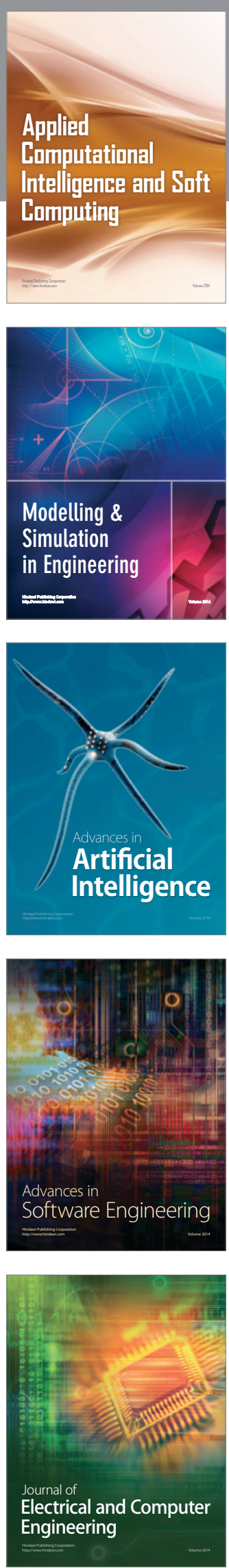